\title{
Finite chemical potential equation of state for QCD from an alternative expansion scheme
}

\author{
Paolo Parotto ${ }^{1, *}$, Szabolcs Borsányi ${ }^{1}$, Zoltan Fodor $^{2,1,3,4}$, Jana N. Guenther ${ }^{5}$, Ruben Kara ${ }^{1}$, \\ Sandor D. Katz ${ }^{6}$, Attila Pásztor $^{6}$, Claudia Ratti ${ }^{7}$, and Kalman K. Szabó ${ }^{1,4}$ \\ ${ }^{1}$ University of Wuppertal, Department of Physics, Wuppertal D-42119, Germany \\ ${ }^{2}$ Pennsylvania State University, Department of Physics, State College, PA 16801, USA \\ ${ }^{3}$ Inst. for Theoretical Physics, ELTE Eötvös Loránd University, Pázmány P. sétány 1/A, H-1117 Bu- \\ dapest, Hungary \\ ${ }^{4}$ Jülich Supercomputing Centre, Forschungszentrum Jülich, D-52425 Jülich, Germany \\ ${ }^{5}$ Aix Marseille Univ., Université de Toulon, CNRS, CPT, Marseille, France \\ ${ }^{6}$ Eötvös University, Budapest 1117, Hungary \\ ${ }^{7}$ Department of Physics, University of Houston, Houston, TX 77204, USA
}

\begin{abstract}
The Taylor expansion approach to the equation of state of QCD at finite chemical potential struggles to reach large chemical potential $\mu_{B}$. This is primarily due to the intrinsic difficulty in precisely determining higher order Taylor coefficients, as well as the structure of the temperature dependence of such observables. In these proceedings, we illustrate a novel scheme [1] that allows us to extrapolate the equation of state of QCD without suffering from the poor convergence typical of the Taylor expansion approach. We continuum extrapolate the coefficients of our new expansion scheme and show the thermodynamic observables up to $\mu_{B} / T \leq 3.5$.
\end{abstract}

\section{Introduction}

The phase diagram of Quantum Chromodynamics (QCD) is a field of major interest from both a theoretical and experimental standpoint. At vanishing baryon density, it is known from lattice QCD [2] that the chiral/deconfinement transition is a smooth crossover. At finite baryon density lattice QCD faces a sign problem, due to the introduction of a real chemical potential that makes the action complex. Although new techniques that allow to directly simulate the theory at finite chemical potential exist [3, 4], they cannot be applied to large scale QCD simulations yet.

Several results have been published that exploit simulations at zero or imaginary chemical potential, and rely on an analytical continuation to extrapolate to real chemical potential. The most straightforward method is the Taylor expansion, whereby the first few $\mu_{B}$-derivatives of thermodynamic quantities are calculated [5,6]. Notable successes of analytic continuation methods include the determination of the QCD transition line, whose $\mu_{B}$-dependence is now known up to the next-to-leading order [7, 8].

At large temperatures, resummed perturbation theory has provided a quantitative description of the chemical potential dependence of several observables, which agree with lattice

\footnotetext{
*Speaker: parotto@uni-wuppertal.de
} 

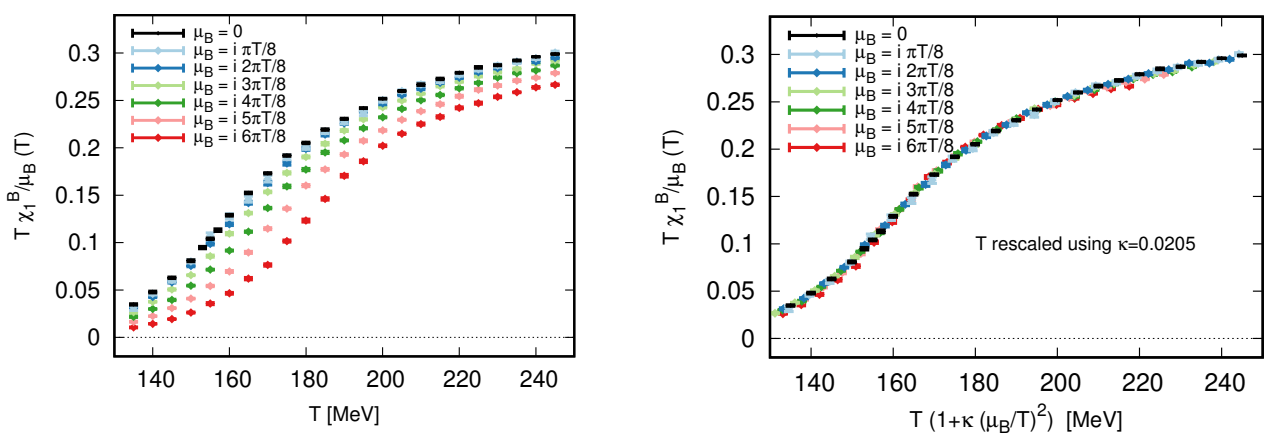

Figure 1. (Left panel): Normalized baryon density at imaginary baryon chemical potentials. The points at $\mu_{B}=0$ (black) show the second baryon susceptibility $\chi_{2}^{B}(T)$. (Right panel): Same curves as in the upper panel, with a temperature rescaled in accordance to Eq. (1) with $\kappa=0.0205$.

calculations $[9,10]$. Similarly, functional methods provide an alternative approach to the study of the phase diagram [11]. However, lattice QCD remains the major first-principle tool of investigation, especially in the transition region of QCD.

From an experimental standpoint, heavy-ion collisions are capable of exploring the phase structure of strongly interacting matter. Systems created in such experiments are modeled through hydrodynamic simulations, for which the equation of state $(\mathrm{EoS})$ of QCD is a crucial input. At vanishing chemical potential it has been known for several years [12-14]. Despite enormous computational efforts aimed at extending these results to higher $\mu_{B}$ by adding more terms in the Taylor series, at the moment even the sixth $\mu_{B}$-derivative of the QCD pressure is only available with modest precision from lattice simulations $[5,6]$.

In this contribution, we describe a new [1], alternative scheme for the extrapolation of the QCD equation of state to finite density, which displays improved convergence over the Taylor series, leading to wider coverage in $\mu_{B}$ and to more precise results for the thermodynamic observables.

\section{The alternative scheme}

We start by observing that, at imaginary chemical potentials, the normalized baryon density $\chi_{1}^{B} / \hat{\mu}_{B}$ presents the same structure at all chemical potentials (including $\hat{\mu}_{B}=0$, where the limit $\lim _{\mu_{B} \rightarrow 0} \chi_{1}^{B} / \hat{\mu}_{B}=\chi_{2}^{B}$ ), as can be seen in the left panel of Fig.1. This is reflected in the fact that a $\mu_{B}$-dependent rescaling of the temperatures makes all the curves collapse onto each other, as shown in the right panel of Fig. 1 . In particular, a single $T$-independent parameter can encode such rescaling quite well according to the following equality:

$$
\frac{\chi_{1}^{B}\left(T, \hat{\mu}_{B}\right)}{\hat{\mu}_{B}}=\chi_{2}^{B}\left(T^{\prime}, 0\right), \quad T^{\prime}=\left(1+\kappa \hat{\mu}_{B}^{2}\right) .
$$

Although quite suggestive, Eq.(1) cannot serve as an alternative expansion scheme as is. Thus, we include higher order terms in the expansion, and allow the expansion parameters to depend on the temperature:

$$
T^{\prime}=T\left(1+\kappa_{2}(T) \hat{\mu}_{B}^{2}+\kappa_{4}(T) \hat{\mu}_{B}^{4}+O\left(\hat{\mu}_{B}^{6}\right)\right) .
$$

In essence, the expansion scheme we present amounts to an expansion in $\Delta T=T^{\prime}-T$ inside the argument of $\chi_{2}^{B}$, and corresponds to a re-organization of the different terms in the 


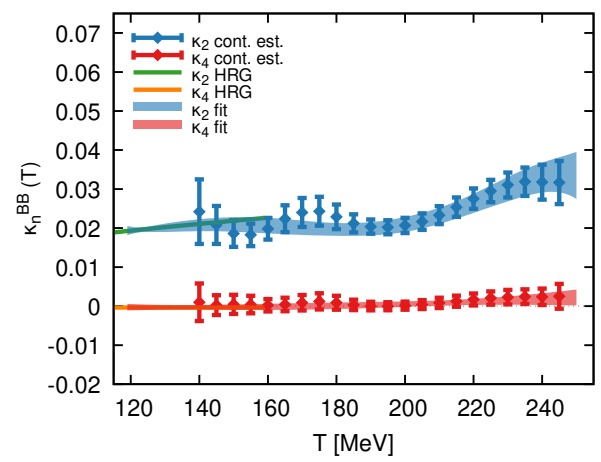

Figure 2. Continuum extrapolated result for the expansion parameters $\kappa_{2}(T)$ and $\kappa_{4}(T)$. HRG results are shown up to $T=160 \mathrm{MeV}$ (in green for $\kappa_{2}$, orange for $\kappa_{4}$, respectively). The bands show correlated polynomial fits.

Taylor series. One can visualize this as an expansion along lines of constant (normalized) baryon density $\chi_{1}^{B} \hat{\mu}_{B}$, while a Taylor expansion is carried out at constant temperature. Working out such expansions, and equating equal order terms, one finds:

$$
\kappa_{2}(T)=\frac{1}{6 T} \frac{\chi_{4}^{B}(T)}{\chi_{2}^{B^{\prime}}(T)}, \quad \kappa_{4}(T)=\frac{1}{360 \chi_{2}^{B^{\prime}}(T)^{3}}\left(3 \chi_{2}^{B^{\prime}}(T)^{2} \chi_{6}^{B}(T)-5 \chi_{2}^{B^{\prime \prime}}(T) \chi_{4}^{B}(T)^{2}\right) .
$$

These relations can be straightforwardly extended to higher orders, and could in principle utilized to determine the coefficients $\kappa_{n}(T)$. However, for $n>2$, such coefficients would still suffer from severe cancellations between competing terms. Thus, exploiting simulations at imaginary chemical potentialts $\hat{\mu}_{B}=i n \pi / 8$, with $n=0, \ldots, 8$, we calculate the proxy quantity:

$$
\Pi\left(T, \hat{\mu}_{B}^{2}\right)=\frac{T^{\prime}-T}{T \hat{\mu}_{B}^{2}}=\kappa_{2}(T)+\kappa_{4}(T) \hat{\mu}_{B}^{2}+O\left(\hat{\mu}_{B}^{4}\right),
$$

at different temperatures, and on three different lattices with $N_{\tau}=10,12,16$. We then perform a combined fit in $\hat{\mu}_{B}^{2}$ and $1 / N_{\tau}^{2}$, from which we extract $\kappa_{2}$ and $\kappa_{4}$ at each temperature. The results for these coefficients are shown in Fig. 2, along with polynomial fits of both $\kappa_{n}(T)$ which take into account the full statistical and systematic correlations between different temperatures. At low temperature, we also show results from the hadron resonance gas (HRG) model, which is in good agreement with our results. Notably, we find a large separation in the values of $\kappa_{2}(T)$ and $\kappa_{4}(T)$, which hints at improved convergence of the expansion.

We reconstruct the baryon density at finite real chemical potential by employing Eq. (1), then obtain the pressure from it by simply integrating over the chemical potential. We show the baryon density and the pressure in the left and right panels of Fig. 3, respectively. We also compare with HRG results at low temperature, finding good agreement at all chemical potentials.

Crucially, the errors are well under control up to $\hat{\mu}_{B}=3.5$, and no unphysical, nonmonotonic behavior is observed. For the baryon density, we compare the results with a simplified scenario in which we set $\kappa_{4} \equiv 0$. We find that the inclusion of a non-zero $\kappa_{4}$ increases the error on the final results, but does not change its prediction. This hints at a better convergence of our series expansion, compared to the Taylor method. 

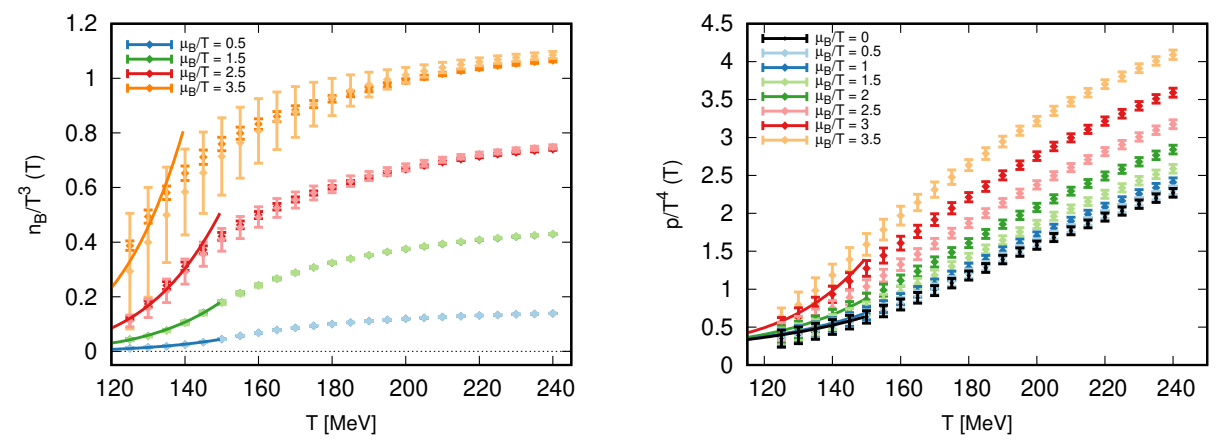

Figure 3. Baryon density (left) and pressure (right) at increasing values of $\hat{\mu}_{B}$. With solid lines we show the results from the HRG model. For the baryon density we also show in darker shades the results obtained by setting $\kappa_{4} \equiv 0$.

\section{Acknowledgments}

This project was funded by the DFG grant SFB/TR55. The project also received support from the BMBF Grant No. 05P18PXFCA. This work was also supported by the Hungarian National Research, Development and Innovation Office, NKFIH grant KKP126769. A.P. is supported by the J. Bolyai Research Scholarship of the Hungarian Academy of Sciences and by the ÚNKP-20-5 New National Excellence Program of the Ministry for Innovation and Technology. The project leading to this publication has received funding from Excellence Initiative of Aix-Marseille University - A*MIDEX, a French "Investissements d'Avenir" programme, AMX-18-ACE-005. This material is based upon work supported by the National Science Foundation under grants no. PHY-1654219 and by the U.S. DoE, Office of Science, Office of Nuclear Physics, within the framework of the Beam Energy Scan Topical (BEST) Collaboration. This research used resources of the Oak Ridge Leadership Computing Facility, which is a DOE Office of Science User Facility supported under Contract DE-AC0500OR22725. The authors gratefully acknowledge the Gauss Centre for Supercomputing e.V. (www.gauss-centre.eu) for funding this project by providing computing time on the GCS Supercomputer HAWK at HLRS, Stuttgart. Part of the computation was performed on the QPACE3 funded by the DFG ind hosted by JSC. C.R. also acknowledges the support from the Center of Advanced Computing and Data Systems at the University of Houston.

\section{References}

[1] S. Borsányi, Z. Fodor, J. N. Guenther, R. Kara, S. D. Katz, P. Parotto, A. Pásztor, C. Ratti and K. K. Szabó, Phys. Rev. Lett. 126, no.23, 232001 (2021) doi:10.1103/PhysRevLett.126.232001 [arXiv:2102.06660 [hep-lat]].

[2] Y. Aoki, G. Endrodi, Z. Fodor, S. D. Katz and K. K. Szabo, Nature 443, 675-678 (2006) doi:10.1038/nature05120 [arXiv:hep-lat/0611014 [hep-lat]].

[3] D. Sexty, Phys. Rev. D 100, no.7, 074503 (2019) doi:10.1103/PhysRevD.100.074503 [arXiv:1907.08712 [hep-lat]].

[4] M. Giordano, K. Kapas, S. D. Katz, D. Nogradi and A. Pasztor, JHEP 05, 088 (2020) doi:10.1007/JHEP05(2020)088 [arXiv:2004.10800 [hep-lat]]. 
[5] S. Borsanyi, Z. Fodor, J. N. Guenther, S. K. Katz, K. K. Szabo, A. Pasztor, I. Portillo and C. Ratti, JHEP 10, 205 (2018) doi:10.1007/JHEP10(2018)205 [arXiv:1805.04445 [heplat]].

[6] A. Bazavov, D. Bollweg, H. T. Ding, P. Enns, J. Goswami, P. Hegde, O. Kaczmarek, F. Karsch, R. Larsen and S. Mukherjee, et al. Phys. Rev. D 101, no.7, 074502 (2020) doi:10.1103/PhysRevD.101.074502 [arXiv:2001.08530 [hep-lat]].

[7] A. Bazavov et al. [HotQCD], Phys. Lett. B 795, 15-21 (2019) doi:10.1016/j.physletb.2019.05.013 [arXiv:1812.08235 [hep-lat]].

[8] S. Borsanyi, Z. Fodor, J. N. Guenther, R. Kara, S. D. Katz, P. Parotto, A. Pasztor, C. Ratti and K. K. Szabo, Phys. Rev. Lett. 125, no.5, 052001 (2020) doi:10.1103/PhysRevLett.125.052001 [arXiv:2002.02821 [hep-lat]].

[9] R. Bellwied, S. Borsanyi, Z. Fodor, S. D. Katz, A. Pasztor, C. Ratti and K. K. Szabo, Phys. Rev. D 92, no.11, 114505 (2015) doi:10.1103/PhysRevD.92.114505 [arXiv:1507.04627 [hep-lat]].

[10] N. Haque and M. Strickland, Phys. Rev. C 103, no.3, 031901 (2021) doi:10.1103/PhysRevC.103.L031901 [arXiv:2011.06938 [hep-ph]].

[11] N. Dupuis, L. Canet, A. Eichhorn, W. Metzner, J. M. Pawlowski, M. Tissier and N. Wschebor, Phys. Rept. 910, 1-114 (2021) doi:10.1016/j.physrep.2021.01.001 [arXiv:2006.04853 [cond-mat.stat-mech]].

[12] S. Borsanyi, G. Endrodi, Z. Fodor, A. Jakovac, S. D. Katz, S. Krieg, C. Ratti and K. K. Szabo, JHEP 11, 077 (2010) doi:10.1007/JHEP11(2010)077 [arXiv:1007.2580 [heplat]].

[13] S. Borsanyi, Z. Fodor, C. Hoelbling, S. D. Katz, S. Krieg and K. K. Szabo, Phys. Lett. B 730, 99-104 (2014) doi:10.1016/j.physletb.2014.01.007 [arXiv:1309.5258 [hep-lat]].

[14] A. Bazavov et al. [HotQCD], Phys. Rev. D 90, 094503 (2014) doi:10.1103/PhysRevD.90.094503 [arXiv:1407.6387 [hep-lat]]. 